\title{
A TYPE OF SINGULAR POINTS FOR A TRANSFORMATION OF
}

\section{THREE VARIABLES*}

B $\mathbf{Y}$

W. V. LOVITT

It is the purpose of this paper to discuss from as geometrical a standpoint as possible the character of a transformation

$$
x=\phi(u, v, w), \quad y=\psi(u, v, w), \quad z=\chi(u, v, w)
$$

near points of a special type at which the jacobian of the transformation vanishes.

Let a particular one of the singular points in question be denoted by $P$, and let $S$ denote the surface through $P$ in the $u v w$-space defined by setting the jacobian of the transformation equal to zero. The point $P$ and the surface $S$ are transformed by (1) into a point $P_{1}$ and surface $S_{1}$ in the $x y z$-space, and the initial assumptions in Section 1 are such that $P$ and $P_{1}$ are non-singular points of $S$ and $S_{1}$, respectively. A neighborhood of the point $P$ is divided by $S$ into two parts. In Section 2 it is shown that under the hypotheses of Section 1 each of these parts is transformed in a one-to-one way into a connected region with interior points in the $x y z$-space, and the two $x y z$-regions so obtained adjoin $S_{1}$ and lie on the same side of that surface. Moreover the single valued inverse functions of $x, y, z$ defined by the transformation in either of the regions adjoining $S_{1}$ are continuous, and they have continuous first derivatives except possibly at points of the surface $S_{1}$.

In the first two sections the transformation is studied in a neighborhood of a particular singular point $P$. But most of the results there found can be extended to apply to a neighborhood of any surface consisting of singular points of the type of $P$. This is done in Section 3. The generalization is analogous to an extension of the well-known theorems on implicit functions due to Bolza. $\dagger$ The methods of Sections 1-3 are applicable to $n$-dimensional spaces with but little more than the necessary changes in notation.

* Presented to the Society, April 2, 1915.

† See his Vorlesungen über Variationrechnung, p. 160; also Mason and Bliss, Fields of extremals in space, these Tr a $\mathrm{n}$ a c t i o n s, vol. 11 (1910), p. 325; or Bliss, Princeton Colloquium Lectures, p. 19. 
In Section 4 we show that the two-parameter family of directions through a point $P$ on the surface $S$, with the exception of a certain critical direction, transforms into a single plane $\pi_{1}$ of directions. This plane $\pi_{1}$ is tangent to the surface $S_{1}$ at the point $P_{1}$. Urner* showed that every plane of directions through $P$ in the $u v w$-space transforms into a single direction in the plane $\pi_{1}$. The direction coincident with instead of distinct from the critical direction $I$ transforms into the two-parameter family of directions through $P_{1}$, with the exception of those in the plane $\pi_{1}$. All curves $D$ having the critical direction and which have the same principal normal are transformed, as Urner has shown, into curves with directions in a common plane $\beta_{1}$. We add that as the radius of first curvature of $D$ varies from infinity through $P$ on the principal normal and out to infinity on the other side again the direction of the transformed curve rotates in $\beta_{1}$ about $P_{1}$ through an angle of 180 degrees.

The character of a transformation in the neighborhood of a point at which the functional determinant vanishes has been the subject of study in a number of papers of comparatively recent date. For a review of the papers in widely different fields bearing on this subject the reader is referred to Chapter II of the Colloquium Lectures referred to above. Bliss showed in these lectures that for a transformation in two variables, regions on opposite sides of the curve defined by the vanishing of the functional determinant of the transformation are transformed into two overlapping regions. The theorems deduced in Sections 1, 2, 4 below afford a generalization of this result to spaces of three or more dimensions. The paper of Urner mentioned in the preceding paragraph contains a discussion of the transformation effected by equations (1) upon the directions of curves through the point $P$, but he does not otherwise discuss the transformation of the region of space adjoining $P$, as is done in the present paper.

\section{The initial assumptions}

Let us consider a real point transformation of three space

$$
x=\phi(u, v, w), \quad y=\psi(u, v, w), \quad z=\chi(u, v, w)
$$

with determinant

$$
J(u, v, w)=\left|\begin{array}{lll}
\phi_{u} & \phi_{v} & \phi_{w} \\
\psi_{u} & \psi_{v} & \psi_{w} \\
\chi_{u} & \chi_{v} & \chi_{w}
\end{array}\right| .
$$

The functions $\phi, \psi, \chi$ are not necessarily analytic but it will be presupposed

* S. E. Urner, Certain singularities of point transformations in space of three dimensions, these Tran s a c t i o n s, vol. 13 (1912), pp. 232-264. 
that

(a) the functions $\phi, \psi, \chi$ are of class $C^{\prime \prime \prime *}$ in a neighborhood of the origin $(u, v, w)=(0,0,0)$;

(b) the following initial conditions are satisfied:

$$
\phi(0,0,0)=\psi(0,0,0)=\chi(0,0,0)=0 ;
$$

(c) $J(0,0,0)=0$;

(d) at the origin $(u, v, w)=(0,0,0)$ at least one of the determinants of the matrix

$$
\mid \begin{array}{lll}
J_{u} & J_{v} & J_{w} \\
\phi_{u} & \phi_{v} & \phi_{w} \\
\psi_{u} & \psi_{v} & \psi_{w} \\
\chi_{u} & \chi_{v} & \chi_{w}
\end{array}
$$

is different from zero.

There is no loss of generality in assuming, as indicated in these conditions, that the singular point $P$ to be considered for the transformation is at the origin in the $u v w$-space, and that the transform of $P$ by (1) is the origin $P_{1}$ in the $x y z$-space. Neither will generality be lost if we assume for convenience that the determinant

$$
H_{1} \equiv\left|\begin{array}{lll}
J_{u} & J_{v} & J_{w} \\
\psi_{u} & \psi_{v} & \psi_{w} \\
\chi_{u} & \chi_{v} & \chi_{w}
\end{array}\right|
$$

is that one of the matrix (2) which does not vanish at the origin. Only a change of notation in $x, y, z, \phi, \psi, \chi$ is required to bring this about if it is not already true.

At least one of the derivatives $J_{u}, J_{v}, J_{w}$ does not vanish at the origin on account of our assumption $(d)$. Hence the equation $J(u, v, w)=0$ can be solved for one of the variables in terms of the other two. There exists therefore a unique surface

$$
u=U(\alpha, \beta), \quad v=V(\alpha, \beta), \quad w=W(\alpha, \beta)
$$

satisfying $J=0$, in which $\alpha$ and $\beta$ are a suitably selected two of the parameters $u, v, w$. Since $J_{u}, J_{v}, J_{w}$ do not all vanish at the origin this point is a nonsingular point of the surface $S$.

* We shall say that a single-valued function $f$ of $(u, v, w)$ is of class $C^{\prime \prime \prime}$ if $f(u, v, w)$ and its partial derivatives of orders one, two, and three are continuous in a region in which $f$ is defined. 
It follows from the derivation of equations $(S)$ above that

$$
J(U, V, W) \equiv 0,
$$

whence

$$
\begin{aligned}
& J_{u} U_{a}+J_{v} U_{a}+J_{w} W_{a}=0, \\
& J_{u} U_{\beta}+J_{v} V_{\beta}+J_{w} W_{\beta}=0 .
\end{aligned}
$$

From these equations we obtain

$$
k J_{u}=\left|\begin{array}{cc}
V_{a} & W_{a} \\
V_{\beta} & W_{\beta}
\end{array}\right|, \quad k J_{v}=\left|\begin{array}{cc}
U_{\beta} & W_{\beta} \\
U_{a} & W_{a}
\end{array}\right|, \quad k J_{w}=\left|\begin{array}{cc}
U_{a} & V_{a} \\
U_{\beta} & V_{\beta}
\end{array}\right|,
$$

where $k$ is a factor of proportionality. Moreover $k \neq 0$ since the surface $S$ is non-singular and consequently the determinants in the second members of equations (4) can not vanish simultaneously.

The transform of the surface $S$ by (1) is a surface

$$
\begin{aligned}
& x=\phi(U, V, W)=X(\alpha, \beta), \\
& y=\psi(U, V, W)=Y(\alpha, \beta), \\
& z=\chi(U, V, W)=Z(\alpha, \beta) .
\end{aligned}
$$

The origin $P_{1}$ is a non-singular point for $S_{1}$ if the determinants of the matrix

$$
\left\|\begin{array}{lll}
X_{a} & Y_{\alpha} & Z_{a} \\
X_{\beta} & Y_{\beta} & Z_{\beta}
\end{array}\right\|
$$

do not vanish simultaneously. But

(6)

$$
\begin{aligned}
\left|\begin{array}{cc}
X_{a} & X_{\beta} \\
Y_{a} & Y_{\beta}
\end{array}\right| & =\left|\begin{array}{ccc}
\phi_{u} U_{a}+\phi_{v} V_{a}+\phi_{w} W_{a} & \phi_{u} U_{\beta}+\phi_{v} V_{\beta}+\phi_{w} W_{\beta} \\
\psi_{u} U_{a}+\psi_{v} V_{a}+\psi_{w} W_{a} & \psi_{u} U_{\beta}+\psi_{v} V_{\beta}+\phi_{w} W_{\beta}
\end{array}\right| \\
& =\left|\begin{array}{ccc}
V_{a} W_{\beta}-V_{\beta} W_{a} & U_{\beta} W_{a}-U_{a} W_{\beta} & U_{a} V_{\beta}-U_{\beta} V_{a} \\
\phi_{u} & \phi_{v} & \phi_{w} \\
\psi_{u} & \psi_{v} & \psi_{w}
\end{array}\right| \\
& =k\left|\begin{array}{lll}
J_{u} & J_{v} & J_{w} \\
\phi_{u} & \phi_{v} & \phi_{v} \\
\psi_{u} & \psi_{v} & \psi_{w}
\end{array}\right|,
\end{aligned}
$$


and by a similar argument

$$
\left|\begin{array}{cc}
X_{a} & X_{\beta} \\
Z_{a} & Z_{\beta}
\end{array}\right|=k\left|\begin{array}{lll}
J_{u} & J_{v} & J_{v} \\
\phi_{u} & \phi_{v} & \phi_{w} \\
\chi_{u} & \chi_{v} & \chi_{w}
\end{array}\right|, \quad\left|\begin{array}{cc}
Y_{a} & Y_{\beta} \\
Z_{a} & Z_{\beta}
\end{array}\right|=k\left|\begin{array}{lll}
J_{u} & J_{v} & J_{w} \\
\psi_{u} & \psi_{v} & \psi_{w} \\
\chi_{u} & \chi_{v} & \chi_{w}
\end{array}\right| \cdot
$$

Thus assumption $(d)$ assures us that $P_{1}$ is an ordinary point on the surface $S_{1}$. We may now state the following theorem.

Theorem I. Under the assumptions $(a),(b),(c),(d)$ the origin $P$ in the uvw-space is a non-singular point of the surface $S$ defined by the equation

$$
J(u, v, w)=0 .
$$

Furthermore in the xyz-space the image $P_{1}$ of the point $P$ by means of equations (1) is a non-singular point of the surface $S_{1}$ into which $S$ is transformed by (1). By our assumptions $(b)$ and $(c)$ the equations

$$
J(u, v, w)=0, \quad y=\psi(u, v, w), \quad z=\chi(u, v, w)
$$

have the initial solution $(u, v, w, y, z)=(0,0,0,0,0)$. The hypothesis (d) justifies the assumption that the determinant (3) is different from zero, as we have seen. Hence by the usual theorems of implicit functions there exists a neighborhood $(0,0,0,0,0)_{\epsilon}{ }^{*}$ in which no two solutions $(u, v, w, y, z)$ of equations (8) have the same projection $(y, z)$, and a neighborhood $(0,0)_{\delta}$ of the point $(y, z)=(0,0)$ in which equations (8) determine $u, v, w$ as functions of class $C^{\prime \prime}$ of $y$ and $z$,

$$
u=u(y, z), \quad v=v(y, z), \quad w=w(y, z)
$$

defining values $(u, v, w, y, z)$ in the neighborhood $(0,0,0,0,0)_{\epsilon}$. By substituting these results in the third of equations (1), a surface

$$
x=X(y, z)
$$

is found, which is the transform by (1) of the surface $S$. This is identical with the surface given by equations $\left(S_{1}\right)$ above.

Let $A_{1}, A_{2}, A_{3} ; B_{1}, B_{2}, B_{3} ; C_{1}, C_{2}, C_{3}$ denote respectively the cofactors of the elements of the first, second, and third rows in the jacobian $J$. Since at the origin

$$
H_{1} \equiv J_{u} A_{1}+J_{v} A_{2}+J_{w} A_{3} \neq 0
$$

* For these theorems see Bliss, Princeton Colloquium Lectures, pp. 8-9. By the notation $(0,0,0,0,0)$. is meant a neighborhood

$$
|u|<\epsilon,|v|<\epsilon,|w|<\epsilon,|y|<\epsilon,|z|<\epsilon
$$

of the point $(0,0,0,0,0)$. 
it follows that $J_{u}, J_{v}, J_{w}$ are not all zero. Moreover some one of the terms $J_{u} A_{1}, J_{v} A_{2}, J_{w} A_{3}$ is different from zero at $P$, let us say $J_{u} A_{1} \neq 0$. Only a change of notation in $u, v, w$ is required to bring this about if it is not already true. Then at the point $P$

$$
A_{1}=\frac{\partial(\psi, \chi)}{\partial(v, w)} \neq 0, \quad J_{u}(0,0,0) \neq 0 .
$$

Thus the tangent plane to the surface $S$ at $P$ is not parallel to the $u$-axis, and the matrix of the determinant $J$ is of rank two at the point $P$.

We now interpret equations (1) as a two-parameter family of curves with the parameters $v, w$. Under the assumption $(d)$, the surface $S_{1}$ is the envelope of the curves (1).* For from equations (8) and (10) the expression

has the factor

$$
\phi_{u}-X_{y} \psi_{u}-X_{z} \chi_{u}
$$

$$
\mid \begin{array}{llll}
0 & J_{\iota} & J_{v} & J_{w} \\
\phi_{u} & \phi_{u} & \phi_{v} & \phi_{w} \\
\psi_{u} & \psi_{\iota} & \psi_{v} & \psi_{u} \\
\chi_{u} & \chi_{u} & \chi_{v} & \chi_{w}
\end{array}=-J J_{u},
$$

which vanishes on the surface $S_{1}$ on account of the first of equations ( $\succ$ ). If we keep $v$ and $w$ fixed and let $u$ vary we get a straight line in the $u v w$-space, and by varying $v$ and $w$ we obtain a two-parameter family of lines parallel to the $u$-axis. These lines are, by the above, transformed by equations (1) into a two-parameter family of curves for which $S_{1}$ is the enveloping surface.

Under the assumptions made, none of the curves of the two-parameter family mentioned cross the surface $S_{1}$, at their points of tangency. For if we put

$$
W(x, y, z) \equiv x-X(y, z)
$$

and in place of $x, y, z$ substitute their values in terms of $u, v, w$ from equations (1), we find

$$
\begin{gathered}
\frac{d W}{d u}=\phi_{u}-X_{y} \psi_{u}-X_{z} \chi_{u}=\frac{1}{H_{1}} J J_{u}, \\
\frac{d^{2} W}{d u^{2}}=\frac{1}{H_{1}}\left(J J_{u u}+J_{u}^{2}\right)+J J_{u} \frac{d}{d u} \frac{1}{H_{1}} .
\end{gathered}
$$

But $d W / d u$ vanishes on the surface $S_{1}$ since $J=0$, while $d^{2} W / d u^{2}$ does not vanish on $S_{1}$ since $J_{u} \neq 0$ by hypothesis. We have thus proved the following

* Mason-Bliss, The properties of curves in space which minimize a definite integral, these Trans a ctions, vol. 9 (1908), pp. 440-466. 
Theorem II. The two-parameter family of straight lines

$$
v=\text { constant }, \quad w=\text { constant }
$$

parallel to the u-axis in the uvw-space is transformed by means of equations (1) into a two-parameter family of curves

$$
x=\phi(u, v, w), \quad y=\psi(u, v, w), \quad z=\chi(u, v, w)
$$

in the xyz-space, with the parameters $v$ and $w$. Under the assumptions (a), (b), $(c),(d)$ this family of curves has an enveloping surface $S_{1}$ which is the transform of the surface $S$ by means of the equations (1). The curves of the family do not cross the surface $S_{1}$ at their points of tangency.

\section{Transformation of a neighborhood of A POINT ON THE singular SURFACE}

In this Section we show that under our assumptions $(a),(b),(c),(d)$ there is a one-to-one correspondence between either of two regions in the uvv-space on opposite sides of the jacobian surface and its transform in the $x y z$-space, the two transformed regions being on the same side of the surface $S_{1}$ which is the transform of the surface $S$ by means of equations (1). Moreover each of the transformed regions is a connected region possessing interior points. The equations of transformation define inverse functions which are continuous at points of the $x y z$-space which are on the surface $S_{1}$, and of class $C^{\prime}$ at points not on $S_{1}$.

The equations

$$
y=\psi(u, v, w), \quad z=\chi(u, v, w)
$$

represent a system of curves in the uvw-space. Every solution $(u, v, w)$ of these equations, when $y$ and $z$ are fixed, determines a value $\xi$ of the determinant $J$ such that equations

$$
J(u, v, w)=\xi, \quad \psi(u, v, w)=y, \quad \chi(u, v, w)=z
$$

are satisfied.

By our assumptions $(b)$ and (c) the equations (12) have the initial solution $(u, v, w, \xi, y, z)=(0,0,0,0,0,0)$. Since the determinant (3) is different from zero the usual theorems of implicit functions justify the statement that there exists a neighborhood $(0,0,0,0,0,0)_{\epsilon}$ in which no two solutions $(u, v, w, \xi, y, z)$ of equations (12) have the same projection $(\xi, y, z)$, and a neighborhood $(0,0,0)_{\delta}$ of the point $(\xi, y, z)=(0,0,0)$ with $\delta \leqq \epsilon$, in which equations (12) determine $u, v, w$ as functions of class $C^{\prime}$ of $\xi, y, z$,

$$
u=u(\xi, y, z), \quad v=v(\xi, y, z), \quad w=w(\xi, y, z) .
$$


If in these equations $\xi$ is placed equal to zero we obtain equations (9) of Section 1.

As a result of the choice of notation mentioned above which assures us that $J_{u}(0,0,0) \neq 0$, the equations of the surface $S$ of Section 1 may now be written in the form

$$
u=U(v, w), \quad v=\alpha, \quad w=\beta .
$$

Consider then in the $u v w$-space a neighborhood

$$
0 \leqq u-U(v, w) \leqq a, \quad|v| \leqq b, \quad|w| \leqq c
$$

of the origin, where $a, b, c$ are so small that the values $(u, v, w, \xi, y, z)$ defined over the region $K$ by equations (12) all lie in $(0,0,0,0,0,0)_{\epsilon}$. Further the region $K$ is to be restricted so that to any $(u, v, w)$ in $K$ there corresponds by (12) a $(\xi, y, z)$ which is in the $\delta$-neighborhood $(0,0,0)_{\delta}$ of the point $(\xi, y, z)=(0,0,0)$. Then there passes through each $(u, v, w)$ in $K$ one and but one of the curves (11). That there is one is seen from equations (12), for to every $(u, v, w)$ there is defined by $(12)$ a $(\xi, y, z)$ in the $\delta$ neighborhood $(0,0,0)_{\delta}$ of $(\xi, y, z)=(0,0,0)$. That there is only one follows from the fact that the only solutions $(u, v, w, \xi, y, z)$ of equations (12) in $(0,0,0,0,0,0)_{\epsilon}$ corresponding to a point $(\xi, y, z)$ in $(0,0,0)_{\delta}$ must satisfy the equations (13). On any one of the curves there is but one point where the determinant $J$ vanishes, namely the point defined by $\xi=0$.

We now substitute the values of $u, v, w$ as given by (13), in equations (12), and by differentiation find that

$$
\begin{aligned}
& J_{u} u_{\xi}+J_{v} v_{\xi}+J_{w} w_{\xi}=1, \\
& \psi_{u} u_{\xi}+\psi_{v} v_{\xi}+\psi_{w} w_{\xi}=0, \\
& \chi_{u} u_{\xi}+\chi_{v} v_{\xi}+\chi_{w} w_{\xi}=0,
\end{aligned}
$$

from which it follows that

$$
u_{\xi}=\frac{1}{H_{1}}\left|\begin{array}{ll}
\psi_{v} & \psi_{w} \\
\chi_{v} & \chi_{w}
\end{array}\right|, \quad v_{\xi}=\frac{1}{H_{1}}\left|\begin{array}{cc}
\psi_{w} & \psi_{u} \\
\chi_{w} & \chi_{u}
\end{array}\right|, \quad w_{\xi}=\frac{1}{H_{1}}\left|\begin{array}{cc}
\psi_{u} & \psi_{v} \\
\chi_{u} & \chi_{v}
\end{array}\right| .
$$

From the first of equations (14) we conclude that the functional determinant $J$ is univariant along the curve (11), and the curve (11) is not tangent to but actually cuts through the surface $S$.

Thus we see that on the curve (11) the functional determinant $J$ has opposite signs on the opposite sides of the surface $S$. In order to fix ideas let us consider $J>0$ on that side of the surface $S$ which contains the positive $u$-axis. Only a change in notation from $u$ to $-u$ is necessary to bring this about if it is not 
already true. The only restriction on the notation $u, v, w$ preceding this was for the purpose of calling $J_{u}$ that one of $J_{u}, J_{v}, J_{w}$ which was not zero.

We propose to show that under the assumptions made, the equations (1) define a one-to-one correspondence between either of the regions

$$
\begin{array}{rlrl}
0 \leqq u-U(v, w) \leqq a, & |v| \leqq b, & |w| \leqq c, \\
-a \leqq u-U(v, w) \leqq 0, & & |v| \leqq b, & |w| \leqq c,
\end{array}
$$

on opposite sides of the surface $S$, and its image $K_{1}$ or $M_{1}$, in the $x y z$-space. We note that according to our assumptions $J=\xi \geqq 0$ in $K$, and $J \leqq 0$ in $M$.

Suppose that the correspondence between $K$ and $K_{1}$ is not one-to-one. It will be shown that this leads to a contradiction with the statement that $J$ varies univariantly on the curve (11). If two points $\left(u^{\prime}, v^{\prime}, w^{\prime}\right)$ and $\left(u^{\prime \prime}, v^{\prime \prime}, w^{\prime \prime}\right)$ in $K$ define the same $(x, y, z)$ they must lie on that one of the curves (11) defined by the values $y, z$, since any $(u, v, w, x, y, z)$ satisfying the original equations (1) determines also a solution $(u, v, w, \xi, y, z)$ of equations (12), and the only solution of equations (12) for a $(u, v, w)$ in $K$ is given by equations (13). They must define distinct values $\xi^{\prime}$ and $\xi^{\prime \prime}$ satisfying $\xi^{\prime} \geqq 0, \xi^{\prime \prime} \geqq 0$ if they lie on the same side $K$ of the surface $S$, since distinct points of a curve (11) can only correspond to distinct values $\xi^{\prime}, \xi^{\prime \prime}$.

The function $\phi$ is continuous with continuous first partial derivatives and takes by hypothesis the same value $x$ on the curve (11) for $\xi=\xi^{\prime}$ and $\xi=\xi^{\prime \prime}$. Hence its derivative for $\xi$ must vanish for at least one value $\xi=\tau$ between $\xi^{\prime}$ and $\xi^{\prime \prime}$. But this derivative is

$$
\frac{d \phi}{d \xi}=\phi_{u} u_{\xi}+\phi_{v} v_{\xi}+\dot{\phi}_{w} w_{\xi} .
$$

With the use of equations (15) we can show that

$$
\frac{d \phi}{d \xi}=\frac{J}{H_{1}} \text {. }
$$

But $\tau$ is positive and $0 \leqq \xi^{\prime}<\tau<\xi^{\prime \prime}$, provided we assume $\xi^{\prime}$ the smaller of $\xi^{\prime}$ and $\xi^{\prime \prime}$, and so the point of the curve (11) defined by $\tau$ is not on the surface $S$. Hence we have indeed reached a contradiction with the statement that $J$ is univariant and vanishes only for $\xi=0$ on the curve (11). Therefore the correspondence between the regions $K$ and $K_{1}$ is one-to-one, and in a similar manner it can be shown that the correspondence between the regions $M$ and $M_{1}$ is one-to-one.

We are now in a position to make some statements with respect to the inverse functions

$$
u=f(x, y, z), \quad v=g(x, y, z), \quad w=h(x, y, z)
$$

defined by equations (1), and the character of the regions $K_{1}$ and $M_{1}$. 
Let us omit from the regions $K$ and $K_{1}$ the points on $S$ and $S_{1}$. Denote the six dimensional set of points so formed by $\kappa$. Then every point $p(u, v$, $u, x, y, z)$ of $\kappa$ is an ordinary point for the equations (1) and satisfies both those equations and the equations (16). By the usual theorems of implicit functions it follows that there exists a neighborhood $p_{\epsilon}$ of $p$ in which no two solutions of equations (1) have the same projection $(x, y, z)$, and a $\delta$-neighborhood of the point $(x, y, z)$ with $\delta \leqq \epsilon$, in which equations (1) determine $u, v, w$ as functions of class $C^{\prime}$ of $x, y, z$. Thus to every point $(u, v, w)$ interior to $K$ there corresponds a point $(x, y, z)$ interior to $K_{1}$ at which $f, g$, $h$ are of class $C^{\prime}$. In like manner we show that the region $M$ contains interior points.

It is evident that $K$ is a connected region. We shall now show that $K$ : is a connected region. To do this it is sufficient to show that any two points $A_{1}, B_{1}$ interior to $K_{1}$ can be joined by a continuous curve also interior to $K_{1}$. Bv equations (16) the points $A_{1}$ and $B_{1}$ are transformed into two points $A$ and $B$ interior to $K$. But $K$ is a connected region, and hence $A$ and $B$ can be joined by a continuous curve interior to $K$, the transform of which by equations (1) is a continuous curve interior to $K_{1}$, joining $A_{1}$ and $B_{1}$, and thus our statement is proved. We may then state the following theorem:

THEOREM III. L'nder the assumptions $(a),(b),(c),(d)$ either of the two regions $K$ and $M$ in the uru-space on opposite sides of the surface $S$ is transformed in a one-to-one way by means of equations (1) into a region, $K_{1}$ or $M_{1}$, in the xyz-space. Each of the regions $K_{1}, M_{1}$ possesses interior points and is a connected region.

From the proof just preceding, the functions $f, g, h$ are of class $C^{\prime}$ in $K_{1}$ or $Y_{1}$ except possibly at points of the surface $S$. We desire to show next that they are continuous even at points which are on the surface $S_{1}$. To do this it is sufficient to show that any infinite sequence of points $q_{i}$ in the region $K_{1}$ with a single limit point $k$ on the surface $S_{1}$, has corresponding to it in the region $K$ an infinite sequence of points $p_{i}$ with a single limit point on the surface $S$, and that this limit point is the point $\pi$ of which $k$ is the image by means of equations (1). The infinite sequence of points $p_{i}$ certainly has at least one condensation point. Let $\pi^{\prime}$ be such a point. Since the functions $\phi, \chi, \psi$ are continuous it follows that the image of $\pi^{\prime}$ must be $k$, and since the correspondence between $K$ and $K_{1}$ is one-to-one $\pi^{\prime}$ coincides with $\pi$. The point $\pi$ necessarily lies upon $S$ since such points are the only ones which can go into the points of $S_{1}$.

We have thus proved that the functions $f, g$, and $h$ are continuous at points of $K_{1}$ or $M_{1}$ on the surface $S_{1}$, and may state the following theorem:

Theorem IV. Under the assumptions $(a),(b),(c),(d)$ the inverse functions

$$
u=f(x, y, z), \quad v=g(x, y, z), \quad u=h(x, y, z)
$$


transforming $K_{1}$ into $K$ (or $M_{1}$ into $M$ ) as described in Theorem $I I I$, and defined by means of equations (1), are continuous at all points of the xyz-space situated in the region $K_{1}$ or on its boundary, and have continuous first derivatives except possibly on the part of the boundary of $K_{1}$ which is coincident with $S_{1}$.

It remains for us to show that the regions on opposite sides of the surface $S$ transform into regions on the same side of the surface $S_{1}$. We take two points $A$ and $B$ within the regions $K$ and $M$, respectively (see Fig. 1), on opposite sides of the surface $S$ and on the same straight line parallel to the $u$-axis through a point $P$ on $S$. The line segment $A P B$ transforms by means
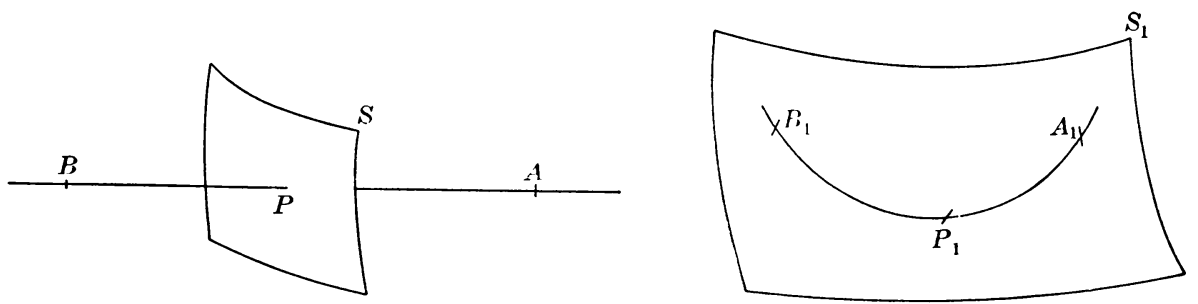

Fig. 1.

of (1) into an arc $A_{1} P_{1} B_{1}$ which by Section 1 is tangent to the surface $S_{1}$ at the point $P_{1}$ and does not cross $S_{1}$ at $P_{1}$. The determinant $J$ does not vanish on the line segments $A B$ except at the point $P$, and hence $P_{1}$ is the only point of $A_{1} P_{1} B_{1}$ which is on the surface $S_{1}$. Thus we see that the points $A_{1}$ and $B_{1}$ are on the same side of the surface $S_{1}$. The point $A$ is in the region $K$ which has been shown to transform in a one-to-one way into the region $K_{1}$ containing the point $A_{1}$, while the point $B$ is in the region $M$ which transforms in a one-to-one way into the region $M_{1}$ containing the point $B_{1}$. But we have just shown that $A_{1}$ and $B_{1}$ are on the same side of the surface $S_{1}$. We may therefore state the following theorem:

Theorem V. Let

$$
x=\phi(u, v, w), \quad y=\psi(u, v, w), \quad z=\chi(u, v, w)
$$

be a transformation which at a point $P$ has properties $(a),(b),(c),(d)$. The surface $S$ on which the jacobian of the transformation vanishes contains $P$ and is

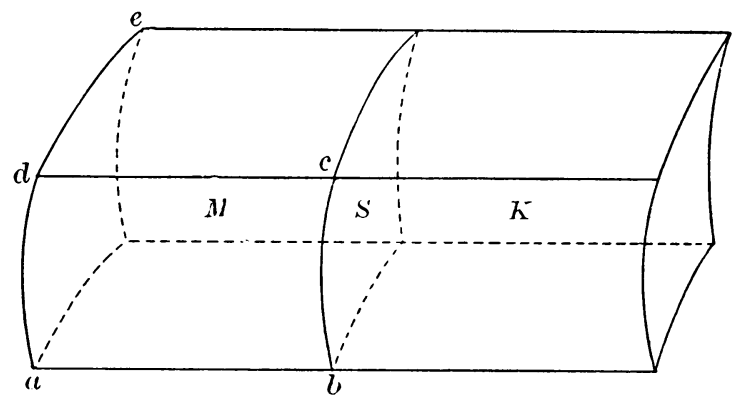

FIG. 2 . 
transformed into a surface $S_{1}$, in the xyz-space containing the image $P_{1}$ of $P$. The portions $K, M$ of the uvw-space on opposite sides of $S$ are transformed in a one-to-one way into portions $K_{1}, M_{1}$ of the xyz-space on the same side of $S_{1}$ (see Fig. 2).

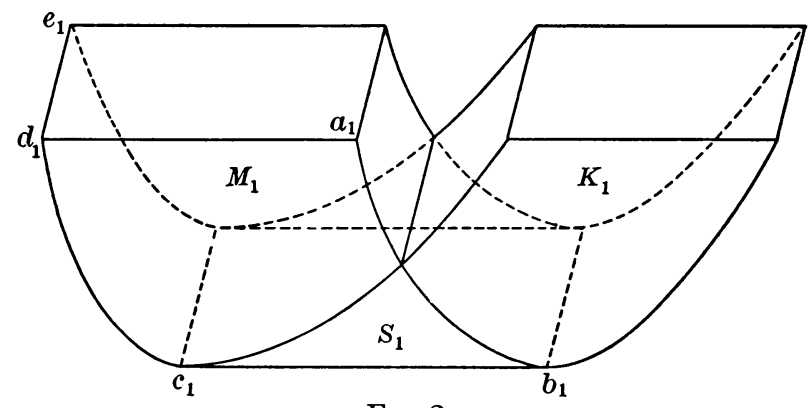

FIG. 2.

3. Transformation of a Neighborhood of a singular surface of any EXTENT

In this section by the use of the assumptions $(a),(b),(c),(d),(e),(f)$ below we extend most of the results of the preceding section to a transformation of a neighborhood of even an extensive part of a singular surface.

Let us now consider the transformation (1) in a neighborhood $S_{\epsilon}$ of the set of points $(u, v, w)$ on the surface

$$
u=U(\alpha, \beta), \quad v=V(\alpha, \beta), \quad w=W(\alpha, \beta)
$$

defined over a finite closed region $\Sigma$ of the $\alpha \beta$-plane.

By a region is understood a piece of the plane which is bounded by a finite number of regular curves. The region is said to be closed if it contains all of its limit points. By a neighborhood $S_{\epsilon}$ is understood the totality of points $(u, v, w)$ for which

$$
|u-U| \leqq \epsilon, \quad|v-V| \leqq \epsilon, \quad|w-W| \leqq \epsilon
$$

when $(\alpha, \beta)$ range over the region $\Sigma$.

It will be presupposed that

(a) the surface $S$ does not intersect itself and has no singular points in $\Sigma$, in other words, the determinants of the matrix

$$
\left\|\begin{array}{lll}
U_{a} & V_{\alpha} & W_{a} \\
U_{\beta} & V_{\beta} & W_{\beta}
\end{array}\right\|
$$

do not vanish simultaneously at any point of $\Sigma$;

(b) the image

$$
\begin{aligned}
& x=\phi(U, V, W)=X(\alpha, \beta), \\
& y=\psi(U, V, W)=Y(\alpha, \beta), \\
& z=\chi(U, V, W)=\stackrel{=}{ }(\alpha, \beta),
\end{aligned}
$$


of the surface $S$ by means of equations (1) does not intersect itself;

(c) the functions $U, V, W$ are of class $C^{\prime}$ in $\Sigma$;

(d) the functions $\phi, \psi, \chi$ are of class $C^{\prime \prime}$ in a neighborhood of the surface $S$;

(e) the functional determinant $J=\partial(\phi, \psi, \chi) / \partial(u, v, w)$ vanishes on the surface $S$;

( $f$ ) at every point of the surface $S$ at least one determinant of the matrix (2) is different from zero.

The assumptions (a) and (b) are sufficient to assure us that there is a one-toone correspondence between the points of the surface $S$ and its image $S_{1}$. For from equations $(S)$, on account of the assumption $(a)$, there is for any point $(u, v, w)$ on $S$ one and but one pair of values of $\alpha, \beta$; and from equations $\left(S_{1}\right)$ by means of the assumption $(b)$ there is for any point $(x, y, z)$ on $S_{1}$ one and but one pair of values of $\alpha, \beta$. Hence for any point $(u, v, w)$ on the surface $S$ there is one and only one point $(x, y, z)$ on the surface $S_{1}$.

Suppose that however small $\epsilon$ is chosen two points in $S_{\epsilon}$ may be found on the same side of $S$ whose images in the $x y z$-space coincide. For points on one side of the surface $S$ the determinant in $(e)$ is positive, while for points on the other side it is negative, since by the assumption $(f)$ one at least of the derivatives $J_{u}, J_{v}, J_{w}$ is different from zero at each point of $S$. Consider now a sequence $\left\{\epsilon_{n}\right\}(n=1,2, \cdots, \infty)$ with limit zero, and the corresponding sequence of regions $\left\{S_{\epsilon_{n}}\right\}$. In every region $S_{\epsilon_{n}}$ there is by hypothesis a pair of distinct points whose images in the $x y z$-space coincide. These two points may be denoted by $P_{n}\left(u_{n}, v_{n}, w_{n}\right), P_{n}^{\prime}\left(u_{n}, v_{n}, w_{n}\right)$ and their image by $\left(x_{n}, y_{n}, z_{n}\right)$. Consider the set of points

$$
p_{n}=\left(u_{n}, v_{n}, w_{n} ; u_{n}^{\prime}, v_{n}^{\prime}, w_{n}^{\prime} ; x_{n}, y_{n}, z_{n}\right)
$$

thinking of $p_{n}$ as a point in a nine-dimensional space. The set $p_{n}(n=1$, $2, \cdots)$ certainly has a condensation point which we designate by $\left(u_{0}, v_{0}, w_{0} ; u_{0}^{\prime}, v_{0}^{\prime}, w_{0}^{\prime} ; x_{0}, y_{0}, z_{0}\right)$, and for which the points $P_{0}\left(u_{0}, v_{0}, w_{0}\right)$ and $P_{0}^{\prime}\left(u_{v}^{\prime}, v_{0}^{\prime}, w_{0}^{\prime}\right)$ necessarily lie upon the surface $S$.

Thus we arrive either at two distinct points $\left(u_{0}, v_{0}, w_{0}\right),\left(u_{0}^{\prime}, v_{0}^{\prime}, w_{0}^{\prime}\right)$ on the surface $S$ whose images in the $x z y$-space coincide; or if $\left(u_{0}, v_{0}, w_{0}\right)$ and $\left(u_{0}^{\prime}, v_{0}^{\prime}, w_{0}^{\prime}\right)$ are the same we arrive at a single limit point on the surface $S$ in every neighborhood of which there are two points whose images in the $x y z-$ space coincide. The first case is impossible since we have shown the one-toone correspondence between the points of the surface $S$ and its image $S_{1}$. "The second case is the one considered in Section 2 and was there shown to be impossible.

Thus we have shown that there must exist a neighborhood $S_{\epsilon}$ in which no two distinct points $(u, v, w)\left(u^{\prime}, v^{\prime}, w^{\prime}\right)$ on the same side of $S$ can define the 
same point $(x, y, z)$ by means of equations (1). It follows that there is a one-to-one correspondence between either one of two suitably chosen neighborhoods on opposite sides of the surface $S$ in the $u v w$-space and its image in the $x y z$-space.

In the same manner as in Section 2 we can now show that a region $K$ in the $u v w$-space consisting of all points of $S_{\epsilon}$ for which $J \geqq 0$, goes by means of equations (1) over into a region $K_{1}$ in the $x y z$-space which contains interior points, and further that the inverse functions $f, g, h$ defined by equations (16) are continuous at every point of $K_{1}$ and of class $C^{\prime}$ except at points on the surface $J_{1}$. Similar statements hold for the region $M$ consisting of all points of $S_{\mathrm{c}}$ for which $J \leqq 0$ and its image $M_{1}$ in the $x y z$-space. We may therefore state the following theorem:

Theorem VI. Let $S$ be a surface of any extent satisfying the hypotheses prescribed at the beginning of this section. Every sufficiently small neighborhood $S_{\epsilon}$ of this surface will be divided into two portions $K$ and $M$ having points of $S$ in common, but in which elsewhere the jacobian $J$ of the transformation (1) has opposite signs. Each of the regions $K$ and $M$ will be transformed in a one-to-one way by the transformation (1) into a region of the xyz-space including the image $S_{1}$ of $S$. In a neighborhood of any point of $S_{1}$ the two xyz-regions $K_{1}$ and $M_{1}$ so defined are both on the same side of $S_{1}$.

\section{The relation of the preceding results to those of Urner}

In a paper referred to above Lirner has considered the transformation by (1) of all the curves

$$
u=a(t), \quad v=b(t), \quad w=c(t)
$$

through a point $P$ at which the functional determinant of the transformation (1) vanishes and is of rank two. His initial assumptions are consequences of our conditions $(c)$ and $(d)$ of Section 2 . It is proposed in this section to study the relationship between the results of the preceding pages and the theorems which Urner has developed.

It is shown in Urner's paper that every curve (18) through $P$, with the exception of those which have a certain critical direction, designated by $I$, is transformed into a curve tangent to a fixed plane $\pi_{1}$. The critical direction through $P$ just mentioned is defined by the ratio $I_{1}: I_{2}: I_{3}$ of the three rows of cofactors of $J$. The direction $a_{1}^{\prime}: b_{1}^{\prime}: c_{1}^{\prime}$ through $P_{1}$ in the xyz-space and corresponding to the direction $a^{\prime}: b^{\prime}: c^{\prime}$ at $P$ of any curve whatsoever of the form (18), always lies in the plane $\pi_{1}$ of directions defined by the equation

$$
A_{i} a_{1}^{\prime}+B_{i} b_{1}^{\prime}+C_{i} c_{1}^{\prime}=0 \text {. }
$$

The plane $\pi_{1}$ is the tangent plane to the surface $S_{1}$ at the point $P_{1}$. For 
from equations (5), (6), (7) of Section 1 we find

$$
A_{i}: B_{i}: C_{i}=H_{1}: H_{2}: H_{3},
$$

where $H_{1}, H_{2}, H_{3}$ define the direction-ratios of the normal to the surface $S_{1}$ at the point $P_{1}$ and are given by the equations

$$
\begin{aligned}
& H_{1} \equiv J_{u} A_{1}+J_{v} A_{2}+J_{w} A_{3}, \\
& H_{2} \equiv J_{u} B_{1}+J_{v} B_{2}+J_{w} B_{3}, \\
& H_{3} \equiv J_{u} C_{1}+J_{v} C_{2}+J_{w} C_{3} .
\end{aligned}
$$

This result permits of ready extension to $n$ variables.

The condition $J(0,0,0)=0$ means that at $P$ the three surfaces

$$
\phi(u, v, w)=0, \quad \psi(u, v, w)=0, \quad \chi(u, v, w)=0
$$

have their normals co-planar. The condition $(d)$, Section 2 , implies that $J$ is of rank 2 ; in particular, if we assume $H_{1} \neq 0$, then the second two surfaces have distinct normals and, by implicit function theory, a well defined curve of intersection. The direction of this curve of intersection is exactly $I_{1}: I_{2}: I_{3}$, and the surface $\phi=0$ is tangent to this curve of intersection at $P$. The assumption $(d)$ means that the direction $I_{1}: I_{2}: I_{3}$ is not tangent to the surface defined by the equation $J=0$.

According to Urner, all directions not coincident with the critical direction, go into directions in a plane $\pi_{1}$. This is the tangent plane to $S_{1}$ at $P_{1}$ (see Fig. 3), as we have shown. According to him also all directions which are not
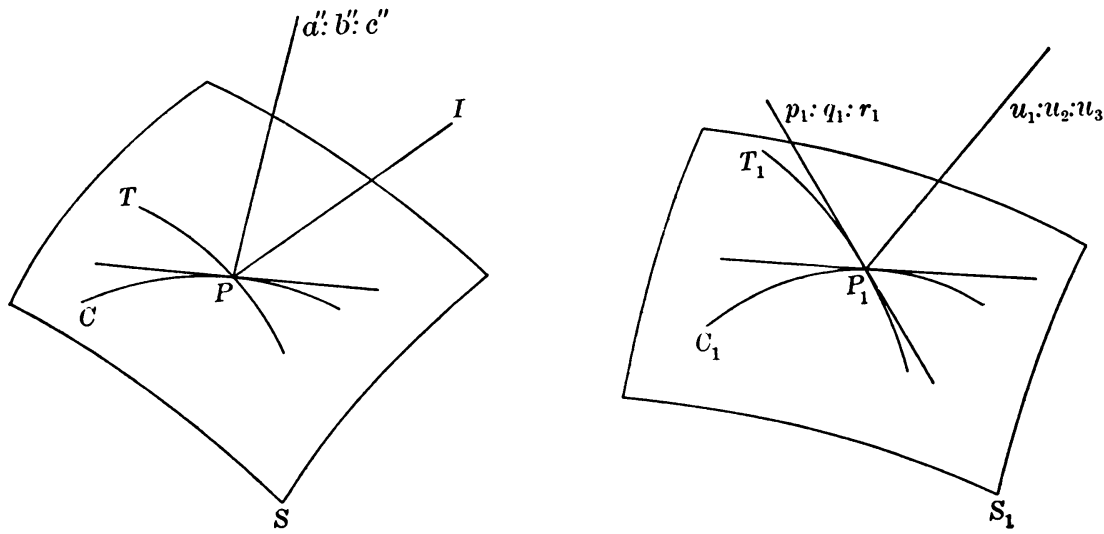

FIG. 3.

coincident with the critical direction $I$, but which are in a common plane $\alpha$ through $I$, go into the same direction tangent to $S_{1}$. Let $\alpha$ be determined by $I$ and the tangent to a curve $C$ on $S$. The curve $C$ goes into a curve $C_{1}$ 
on $S_{1}$, the direction of $C$ at $P$ being transformed into that of $C_{1}$ at $P_{1}$, and it follows that all directions in $\alpha$ go into the tangent to $C_{1}$ on $S_{1}$.

Take now a curve $D$ in the $u v w$-space with initial direction coincident with instead of distinct from the critical direction $I$, with equations of the form (18), and with the length of arc as the parameter $t$, and furthermore let the numbers $I_{1}, I_{2}, I_{3}$ be modified by a common factor if necessary so that they are direction cosines. Then at $P, a^{\prime}=I_{1}, b^{\prime}=I_{2}, c^{\prime}=I_{3}$. Further, since $t$ is the length of arc, the values $a^{\prime \prime}, b^{\prime \prime}, c^{\prime \prime}$ define the principal normal perpendicular to the direction $I$, and this direction goes into a direction

$$
\begin{aligned}
& p_{1}=\phi_{u} a^{\prime \prime}+\phi_{v} b^{\prime \prime}+\phi_{w} c^{\prime \prime}, \\
& q_{1}=\psi_{u} a^{\prime \prime}+\psi_{v} b^{\prime \prime}+\psi_{w} c^{\prime \prime}, \\
& r_{1}=\chi_{u} a^{\prime \prime}+\chi_{v} b^{\prime \prime}+\chi_{w} c^{\prime \prime},
\end{aligned}
$$

in the $x y z$-space. In Urner's equation (9) the constant $k$ is unity under these circumstances, and these equations take the form

$$
a_{1}^{\prime \prime}=p_{1}+U(\phi, I), \quad b_{1}^{\prime \prime}=q_{1}+U(\psi, I), \quad c_{1}^{\prime \prime}=r_{1}+U(\chi, I),
$$

where

$$
U(F, I) \equiv I_{1}^{2} F_{u u}+I_{2}^{2} F_{v v}+I_{3}^{2} F_{w w}+2 I_{2} I_{3} F_{v w}+2 I_{3} I_{1} F_{w u}+2 I_{1} I_{2} F_{u v} \text {. }
$$

All of the curves $D$ of the type just described which have the same principal normal are transformed into curves with coplanar directions, as Urner has shown. For if two curves $D$ and $\bar{D}$ have the same principal normal, then $\bar{a}^{\prime \prime}=\lambda a^{\prime \prime}, \bar{b}^{\prime \prime}=\lambda b^{\prime \prime}, \bar{c}^{\prime \prime}=\lambda c^{\prime \prime}$. Hence for the transform $\bar{D}_{1}$ of $\bar{D}$ by equations (1)

$$
\bar{a}_{1}^{\prime \prime}=\lambda p_{1}+U(\phi, I), \quad \bar{b}_{1}^{\prime \prime}=\lambda q_{1}+U(\psi, I), \quad \bar{c}_{1}^{\prime \prime}=\lambda r_{1}+U(\chi, I) .
$$

We get the directions of the transforms of all curves $\bar{D}$ with the same principal normal (i. e., the same osculating plane) as $D$ by letting $\lambda$ vary, and we see with Urner that the transformed directions $\bar{a}_{1}^{\prime \prime}: \bar{b}_{1}^{\prime \prime}: \bar{c}_{1}^{\prime \prime}$ all lie in the plane $\beta_{1}$ determined by $p_{1}: q_{1}: r_{1}$ and

$$
U(\phi, I): U(\psi, I): U(\chi, I)=U_{1}: U_{2}: U_{3},
$$

thought of as directions through $P_{1}$.

To get this plane geometrically take the plane through $I$ and $a^{\prime \prime}: b^{\prime \prime}: c^{\prime \prime}$ in the $u v w$-space. It cuts a curve $T$ out of the surface $S$ (see Fig. 3 ) which in turn determines a curve $T_{1}$ on $S_{1}$ with direction $p_{1}: q_{1}: r_{1}$. The plane $\beta_{1}$ required is that through $p_{1}: q_{1}: r_{1}$ and $U_{1}: U_{2}: U_{3}$. As $\lambda$ varies from $-\infty$ to $+\infty$, i. e., as the radius of first curvature of $D$ varies from infinity through $P$ on the line whose direction cosines are $a^{\prime \prime}: b^{\prime \prime}: c^{\prime \prime}$, and out to infinity on the other side again, the direction $\bar{a}_{1}^{\prime \prime}: \bar{b}_{1}^{\prime \prime}: \bar{c}_{1}^{\prime \prime}$ starts at $-p_{1}:-q_{1}:-r_{1}$ and rotates in $\beta_{1}$ about $P_{1}$ to $p_{1}: q_{1}: r_{1}$, passing through $U_{1}: U_{2}: U_{3}$ for $\lambda=0$. 